\title{
On end degrees and infinite cycles in locally finite graphs
}

\author{
Henning Bruhn Maya Stein
}

\begin{abstract}
We introduce a natural extension of the vertex degree to ends. For the cycle space $\mathcal{C}(G)$ as proposed by Diestel and Kühn [4, 5], which allows for infinite cycles, we prove that the edge set of a locally finite graph $G$ lies in $\mathcal{C}(G)$ if and only if every vertex and every end has even degree. In the same way we generalise to locally finite graphs the characterisation of the cycles in a finite graph as its 2-regular connected subgraphs.
\end{abstract}

\section{Introduction}

The cycle space of a finite graph is the $\mathbb{Z}_{2}$-vector space consisting of all sums of edge sets of cycles. In its naive extension to infinite graphs, where the elements of the cycle space are finite edge sets, many theorems known for finite graphs become false. Recent studies, see Diestel [2] for an introduction and a survey, strongly suggest that these issues arise because in infinite graphs, certain 'infinite cycles' should be allowed. This idea leads to a definition of the cycle space $\mathcal{C}(G)$ for locally finite graphs $G$, in which the cycles are those subgraphs of $G$ whose closure in $|G|$, the compactification of $G$ by its ends, is a circle, i.e. a homeomorphic image of the unit circle. We will introduce $\mathcal{C}(G)$, which has been proposed by Diestel and Kühn [4,5], formally in the next section.

Using $\mathcal{C}(G)$, almost all properties of the cycle space of a finite graph can be extended to locally finite graphs. Yet, to date there is no generalisation of one of the most basic characterisations of the elements of the cycle space:

Theorem 1. Let $H$ be a subgraph of a finite graph $G$. Then $E(H)$ is an element of the cycle space of $G$ if and only if every vertex of $G$ has even degree in $H$.

Simple examples show that for infinite graphs it is not sufficient to consider vertex degrees. Consider, for instance, the double ray $D$. Since $|D|$ is homeomorphic to the unit interval, it does not contain any circles, hence, it follows that $\mathcal{C}(D)=\{\emptyset\}$. Thus $E(D) \notin \mathcal{C}(D)$, even though every vertex of $D$ has degree 2 in $D$. The problem here seems to arise from the ends rather than the vertices of the considered graph. Diestel and Kühn [5] raised the following problem:

Problem 2. Characterise the circles and the elements of the cycle space of an infinite graph in purely combinatorial terms, such as vertex degrees and 'degrees of ends'.

We introduce a concept of end degrees that is a natural extension of vertex degrees; instead of incident edges we count edge-disjoint rays (for degrees in 
subgraphs it will be necessary to substitute 'rays' with 'arcs'). This notion allows us to solve the first part of Problem 2. We prove a straightforward adaption of the well-known fact that the cycles in a finite graph are exactly its 2-regular connected subgraphs.

Proposition 3. Let $C$ be a subgraph of a locally finite graph $G$. Then $\bar{C}$ is a circle if and only if $\bar{C}$ is topologically connected and every vertex or end $x$ of $G$ with $x \in \bar{C}$ has degree two in $C$.

Depending on its degree, an end can be assigned a parity, i.e. the label 'even' or 'odd' - as long as it has finite degree. Inspired by Laviolette [10], who introduced a concept to measure the parity of vertices of infinite degree, we assign a parity also to ends of infinite degree. A classification of ends into even and odd ends has already been achieved by Nash-Williams [12] for the case of eulerian graphs with only finitely many ends. Our definition coincides with Nash-Williams' in these graphs but covers all locally finite graphs. Moreover, with our definition the following important special case of Problem 2 becomes true, which is the main result of this paper.

Theorem 4. Let $G$ be a locally finite graph. Then $E(G) \in \mathcal{C}(G)$ if and only if every vertex and every end of $G$ has even degree.

An extension of this characterisation to arbitrary subgraphs of $G$ would solve Problem 2 completely. We shall offer a conjecture in that respect (see Section 4).

The paper is organised as follows. After some basic definitions in Section 2, we introduce and discuss our end degree concept in Sections 3 and 4 . Theorem 4 will be proved in Section 5. In Section 6, we prove Proposition 3 and some other results, and in the last section we briefly discuss an alternative notion of parity.

\section{Definitions}

The basic terminology we use can be found in Diestel [3]. Let $G=(V, E)$ be a fixed locally finite graph. A 1-way infinite path in $G$ is called a ray, a 2 -way infinite path is a double ray, and the subrays of a ray or double ray are its tails. Two rays in $G$ are equivalent if no finite set of vertices separates them; the corresponding equivalence classes of rays are the ends of $G$. We denote the set of these ends by $\Omega=\Omega(G)$. A ray $R$ in an end $\omega$ will also be called an $\omega$-ray.

Let us define a topology on $G$ together with its ends, which is known as the Freudenthal compactification of $G$. We begin by endowing $G$ itself (without ends) with the usual topology of a 1-complex. (Thus, every edge is homeomorphic to the real interval $[0,1]$, and the basic open neighbourhoods of a vertex $v$ are the unions of half-open intervals $[v, z)$, one for every edge $e$ at $v$ with $z$ an inner point of e.) In order to extend this topology to $G \cup \Omega$, we take as a basis of open neighbourhoods of a given end $\omega \in \Omega$ the sets of the form

$$
\hat{C}(S, \omega):=C(S, \omega) \cup \Omega(S, \omega) \cup \stackrel{\circ}{E}(S, \omega),
$$

where $S \subseteq V$ is a finite set of vertices, $C(S, \omega)$ is the (unique) component of $G-S$ in which every ray from $\omega$ has a tail, $\Omega(S, \omega)$ is the set of all ends $\omega^{\prime} \in \Omega$ whose rays have a tail in $C(S, \omega)$, and $\stackrel{\circ}{E}(S, \omega)$ is the set of inner points of edges between $S$ and $C(S, \omega)$. Let $|G|$ denote the corresponding topological space on 
the point set $V \cup \Omega \cup \cup E$. We shall freely view $G$ and its subgraphs either as abstract graphs or as subspaces of $|G|$. We note that $|G|$ is a Hausdorff space. Later on, we shall need the following fact.

Theorem 5 (Diestel and Kühn [6]). When a graph $G$ is locally finite, every closed connected subset of $|G|$ is path-connected.

A continuous image of the unit interval $[0,1]$ in $|G|$ is a topological path. The images of 0 and 1 are the endpoints of the topological path. A homeomorphic image of $[0,1]$ in $|G|$ is called an arc in $|G|$. Analogously to $\omega$-rays, let us say that an arc is an $\omega$-arc if the end $\omega$ is one of its endpoints. The following lemma can be found in Hall and Spencer [9, p. 208].

Lemma 6. Every topological path with distinct endpoints $x, y$ in a Hausdorff space $X$ contains an arc between $x$ and $y$.

A set $C \subseteq|G|$ is a circle if it is homeomorphic to the unit circle. Then $C$ includes every edge of which it contains an inner point, and the graph consisting of these edges and their endvertices is the cycle defined by $C$. Conversely, it is not hard to show [4] that $C \cap G$ is dense in $C$, so every circle is the closure in $|G|$ of its cycle and hence defined uniquely by it. Note that every finite cycle in $G$ is also a cycle in this sense, but there can also be infinite cycles; see [2] for examples. The edge set of a cycle is called a circuit.

Call a family $\left(D_{i}\right)_{i \in I}$ of subsets of $E$ thin if no edge lies in infinitely many of the $D_{i}$. Let the sum $\sum_{i \in I} D_{i}$ of this family be the set of all edges that lie in $D_{i}$ for an odd number of indices $i$, and let the cycle space $\mathcal{C}(G)$ of $G$ be the set of all sums of (thin families of) circuits, finite or infinite. Symmetric difference as addition makes of $\mathcal{C}(G)$ a $\mathbb{Z}_{2}$-vector space, which coincides with the usual cycle space of $G$ when $G$ is finite. We remark that $\mathcal{C}(G)$ is itself closed under taking infinite thin sums $[4,5]$, which is not obvious from the definitions.

The following theorem will serve as our main tool to decide whether a graph is an element of its own cycle space, namely if and only if it contains no odd cut.

Theorem 7 (Diestel and Kühn [4]). Let $G$ be a locally finite graph, and let $Z \subseteq E(G)$. Then $Z \in \mathcal{C}(G)$ if and only if $|F \cap Z|$ is even for every finite cut $F$ of $G$.

A finite cut $F$ of $G$ separates a set $S \subseteq V(G)$ from an end $\omega \in \Omega$ if it meets every ray of $\omega$ that starts in $S$. This is equivalent to that the (unique) component $C$ of $G-F$ with $\omega \in \bar{C}$ is disjoint from $S$. Similarily, $F$ separates two ends $\omega$ and $\omega^{\prime}$, if the closure of each component of $G-F$ contains at most one of $\omega, \omega^{\prime}$.

If $C$ is a subgraph of $G$, write $\partial_{G} C$ or, where no confusion is possible, $\partial C$, for the cut $E_{G}(C, G-C)$. Call a connected induced subgraph $C$ of $G$ a region, if the cut $\partial C$ is finite. The region $C$ is even resp. odd if $|\partial C|$ is even resp. odd.

\section{End degrees and parity}

As ends are equivalence classes of rays, the degree of an end should in some way be related to its rays. Also, the rays may be seen as somewhat analogous to the 
incident edges of a vertex, whose number is the degree of the vertex. Therefore, as a first try, we might count the maximal number of disjoint rays in an end (sometimes called the multiplicity of the end). For this notion of an end degree, however, Theorem 4 fails as the graph $G$ in Figure 1 demonstrates. Since $G$ contains an odd cut, its edge set is not an element of $\mathcal{C}(G)$, by Theorem 7 . But each vertex degree is even, and the maximal number of disjoint rays in each end is two.

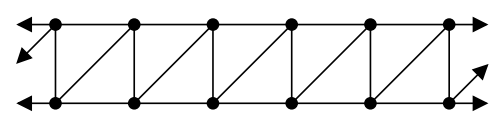

Figure 1: The multiplicity of each end is even, but $E(G) \notin \mathcal{C}(G)$.

Looking more closely we see that although the maximal number of disjoint rays in each end is even, the maximal number of edge-disjoint rays is odd, namely three. Thus with this measure instead we would have correctly decided that $E(G) \notin \mathcal{C}(G)$. Formally, let us define the degree of an end $\omega$ in a graph $G$ as

$$
d(\omega):=\sup \{|\mathcal{R}|: \mathcal{R} \text { is a set of edge-disjoint } \omega \text {-rays }\} \in \mathbb{N} \cup\{\infty\} .
$$

Although we will not use this, we remark that it is not difficult to prove that the supremum is attained, i.e. if $d(\omega)=\infty$, then there exists an infinite set of edge-disjoint $\omega$-rays. Andreae [1] proves a similar result.

Our degree concept clearly divides the ends of finite degree into even and odd ends, but how are we to deal with ends of infinite degree? We may not simply treat them as odd ends, since the edge set of the infinite grid obviously is an element of its cycle space but the only end of the grid has infinite degree.

On the other hand, classifying all ends of infinite degree as even is not any better: consider the graph $G$ in Figure 2. All vertex degrees are even and both ends have infinite degree, but $G$ has an odd cut (which by Theorem 7 implies that $E(G) \notin \mathcal{C}(G))$.

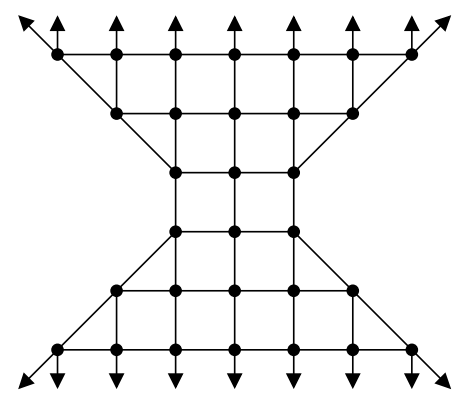

Figure 2: Both ends have infinite degree, but $E(G) \notin \mathcal{C}(G)$.

Consequently, the degree, if infinite, is not a sufficiently fine notion to determine the parity of an end. For an adequate refinement we will use the following characterisation of ends with even finite degree. 
Lemma 8. In a locally finite graph $G$ let $\omega \in \Omega(G)$ have finite degree $k$. Then the following statements are equivalent:

(i) $k$ is even;

(ii) there is a finite $S \subseteq V(G)$ such that for every finite set $S^{\prime} \supseteq S$ of vertices the maximal number of edge-disjoint $\omega$-rays starting in $S^{\prime}$ is even.

Proof. Consider a set $\mathcal{R}$ of edge-disjoint $\omega$-rays of maximal cardinality $|\mathcal{R}|=k$, and let $U$ be the set of starting vertices of $\mathcal{R}$. Then, for every finite set $S^{\prime} \supseteq U$, $\mathcal{R}$ has maximal cardinality among all sets of edge-disjoint $\omega$-rays starting in $S^{\prime}$. Thus, putting $S:=U$, we deduce that (i) implies (ii). Also, (ii) implies (i), which we see by choosing $S^{\prime}=S \cup U$.

As every finite set $S \subseteq V(G)$ gives (essentially) rise to a neighbourhood $\hat{C}(S, \omega)$ of $\omega$, condition (ii) in Lemma 8 can be alternatively formulated using these neighbourhoods, or using regions whose closures contain $\omega$ :

(ii') There is a region $A$ of $G$ with $\omega \in \bar{A}$ such that for every region $B \subseteq A$ of $G$ with $\omega \in \bar{B}$ the maximal number of edge-disjoint rays of $\omega$ starting outside $B$ is even.

So we call an end $\omega$ of a locally finite graph even if $\omega$ satisfies (ii) of Lemma 8 . Otherwise $\omega$ is odd. Thus, $\omega$ is odd if and only if for all finite $S \subseteq V(G)$ there is a finite set $S^{\prime} \supseteq S$ such that the maximal number of edge-disjoint $\omega$-rays starting in $S^{\prime}$ is odd. By Lemma 8 , an end $\omega$ of finite degree is even if and only if $d(\omega)$ is even.

Observe that our notion of parity is not symmetric. Roughly speaking, while for an even end $\omega$ any maximal set of $\omega$-rays starting in any 'close enough' $S$ has even cardinality, an odd end $\omega^{\prime}$ can only guarantee that there are arbitrarily 'close' sets $S$ in which start odd maximal sets of $\omega^{\prime}$-rays. In other words, the quantifiers are exchanged: they read $\exists S \forall S^{\prime} \supseteq S \ldots$ for an even end, and $\forall S$ $\exists S^{\prime} \supseteq S \ldots$ for an odd end. We will return to this issue in Section 7 .

Let us turn back to the examples that motivated our struggle for a concept of parity, the infinite grid, and the graph $G$ in Figure 2. Their ends turn out to have the expected even resp. odd degree. Indeed, for the infinite grid we can choose $S=\emptyset$, and for $G$ it suffices for $S$ to separate the two ends of $G$. Then $|E(S, C)|$ is odd for any infinite component $C$ of $G-S$, and so is $\left|E\left(S^{\prime}, C^{\prime}\right)\right|$ for any $S^{\prime} \supseteq S$ and infinite component $C^{\prime}$ of $G-S^{\prime}$ (because all vertex degrees are even).

\section{Degrees and parity in subgraphs}

It is not possible to extend our degree notion literally to subgraphs $H$ of $G$. There are two obstacles.

First, we cannot simply measure the degrees of the ends of $H$ (as opposed to those of $G$ ). This is not surprising as $H$ is embedded in the space $|G|$. If $H$ is a double ray, for instance, then (viewed as a graph on its own and not as a subgraph) it has two ends, each of which has degree 1 . On the other hand, the tails of $H$ may lie in the same end of $G$, in which case $\bar{H}$ is a circle in $|G|$. Thus the ends contained in $\bar{H}$ should have degree 2 in $H$, not 1 . Therefore, we only consider ends of $G$ (and not of $H$ ). 
Second, even taking that into account, the literal extension to subgraphs fails: Consider the bold subgraph of the graph in Figure 3, and let $\omega$ be the end of $G$ "to the right", i.e. that contains the ray at the bottom. Then, apart from tail-equivalence, $\omega$ has only one ray in $H$, that is, the maximal number of edge-disjoint $\omega$-rays in $H$ is 1 . But as $\bar{H}$ is a circle, we would expect the end $\omega$ to have degree 2 in $H$. In contrast, if we consider the maximal number

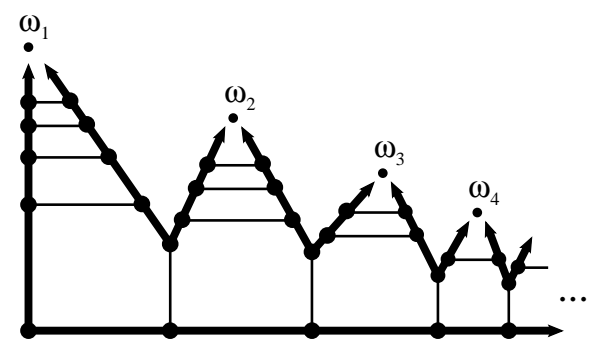

Figure 3: In subgraphs, counting edge-disjoint rays is not enough.

of edge-disjoint $\omega$-arcs in $\bar{H}$ instead of counting edge-disjoint $\omega$-rays in $H$ we obtain the desired degree 2 , as $\omega$ is the endpoint not only of the ray at the bottom, but also of a disjoint arc going through the ends $\omega_{1}, \omega_{2}, \ldots$. Counting arcs will indeed turn out to be successful, and the following proposition shows that in $G$ it makes actually no difference whether we count rays or arcs:

Proposition 9. Let $G$ be a locally finite graph, and let $\omega$ be an end of $G$. Then for every finite set $S \subseteq V(G)$ the maximal number of edge-disjoint $\omega$-rays starting in $S$ equals the maximal number of edge-disjoint $\omega$-arcs starting in $S$.

For its proof we need the following lemma, which can be proved in a similar fashion as a corresponding result by Halin [8] for disjoint (rather than edgedisjoint) rays:

Lemma 10. Let $G$ be a locally finite graph, let $\omega \in \Omega(G)$, and let $S \subseteq V(G)$ be finite. Then the maximal number of edge-disjoint rays in $\omega$ starting in $S$ equals the minimum cardinality of a cut separating $S$ from $\omega$.

Proof of Proposition 9. By Lemma 10, the maximal number of edge-disjoint rays in $\omega$ starting in $S$ equals the minimal cardinality of a finite cut $F$ that separates $S$ from $\omega$. Since $F$ is finite every $\omega$-arc starting in $S$ needs to use one of the edges in $F$. Therefore, the number of $\omega$-arcs starting in $S$ cannot be higher than the number of rays in $\omega$ starting in $S$.

Hence, for a subgraph $H$ of a locally finite graph $G$, and $\omega \in \Omega(G)$, we define, analogously to the definition of $d(\omega)$ given above, the degree of $\omega$ in $H$ as

$$
d_{H}(\omega):=\sup \{|\mathcal{R}|: \mathcal{R} \text { is a set of edge-disjoint } \omega \text {-arcs in } \bar{H}\} \in \mathbb{N} \cup\{\infty\} \text {. }
$$

We note that the supremum is attained, a fact that we will, however, neither make use of nor prove. Furthermore, observe that $d(\omega)=d_{G}(\omega)$. Indeed, suppose otherwise, i.e. $d(\omega)<d_{G}(\omega)$. So, in particular, $d(\omega)$ is finite. For a 
set of $d(\omega)+1$ edge-disjoint $\omega$-arcs, let $S \subseteq V(G)$ be a choice of exactly one vertex from each of the arcs. Then, by Proposition 9, there are also $d(\omega)+1$ edge-disjoint $\omega$-rays starting in $S$, a contradiction.

It is possible to prove a version of Lemma 10 for subgraphs and $\omega$-arcs. Then the following two characterisations of end degrees in terms of cut cardinalities are immediate. For the sake of brevity, and since for the proof of our main result we only need Corollary 12 for $H=G$ (in which case the corollary follows from Lemma 10 in the version we proved), we will not provide the subgraph version of Lemma 10.

Corollary 11. Let $G$ be a locally finite graph, let $H$ be a subgraph, and let $\omega \in \Omega(G)$. Then $d_{H}(\omega)=k \in \mathbb{N}$ if and only if $k$ is the smallest integer such that every finite $S \subseteq V(G)$ can be separated from $\omega$ with a finite cut that shares exactly $k$ edges with $E(H)$.

Corollary 12. Let $G$ be a locally finite graph, let $H$ be a subgraph, and let $\omega \in \Omega(G)$. Then $\omega$ has even degree in $H$ if and only if there is a finite $S \subseteq V(G)$ such that for every finite $S^{\prime} \subseteq V(G)$ with $S^{\prime} \supseteq S$ it holds: if $F \subseteq E(G)$ is a finite cut separating $S^{\prime}$ and $\omega$ with $|F \cap E(H)|$ minimal, then $|F \cap E(H)|$ is even.

Analoguos to the degree, the parity of an end in $H$ is defined with arcs:

Definition 13. An end $\omega$ of $G$ is even in $H$ if there is a finite $S \subseteq V(G)$ such that for every finite $S^{\prime} \subseteq V(G)$ with $S^{\prime} \supseteq S$ the maximal number of edge-disjoint $\omega$-arcs in $\bar{H}$ starting in $S^{\prime}$ is even. Otherwise, $\omega$ is odd in $H$.

Note that by Proposition 9, the definition of parity is consistent with the one given previously. Moreover, it can be seen similarly as in the proof of Lemma 8 that for an end $\omega$ with finite degree in $H, \omega$ has even degree in $H$ if and only if $d_{H}(\omega)$ is even.

Problem 2 seeks a characterisation of the cycle space elements in 'purely combinatorial terms'. Although our notion of degree and parity of an end in a subgraph is based on topological concepts, Corollaries 11 and 12 give a combinatorial descripition of both degree and parity. Hence an analogue of Theorem 4 for subgraphs $H$ of $G$ would yield a complete solution of Problem 2. The forward direction of such an analogue can be proved easily with the same methods as used for Theorem 4. Furthermore, for graphs with only countably many ends the problem is solved by Proposition 20. In view of this, and in view of Theorems 3 and 4, and two more results in Section 6, which demonstrate that end degree and parity behave in many aspects similar to vertex degree and parity in finite graphs, we offer the following conjecture:

Conjecture 14. Let $H$ be a subgraph of a locally finite graph $G$. Then $E(H) \in$ $\mathcal{C}(G)$ if and only if every vertex and every end has even degree in $H$.

\section{$5 \quad$ Proof of Theorem 4}

The forward direction follows from Theorem 7, which ensures that every finite cut of $G$ is even, and thus together with Corollary 12 implies the assertion. 
For the backward direction suppose that $E(G) \notin \mathcal{C}(G)$. Observe that we may assume $G$ to be connected, which means in particular that $G$ is countable. We shall find a sequence $C_{1} \supseteq C_{2} \supseteq \ldots$ of regions of $G$ that satisfy

(i) $\partial_{G} C_{n}$ is an odd cut, for $n \geq 1$;

(ii) $C_{n} \cup N\left(C_{n}\right) \subseteq C_{n-1}$, for $n \geq 2$; and

(iii) if $D$ is a region of $G$ with $C_{n-1} \supseteq D \supseteq C_{n}$ then $\left|\partial_{G} D\right| \geq\left|\partial_{G} C_{n-1}\right|$, for $n \geq 2$,

Then $G$ has an odd end, contradicting the assumption, as desired. Indeed, it is easy to see that there is a ray that has a tail in every $C_{n}$; denote by $\omega$ the corresponding end. Consider any finite $S \subseteq V(G)$, and choose $I$ large enough such that $C_{I} \subseteq C(S, \omega)$, which is possible by (ii). By applying Menger's theorem (in the line graph version) between $\partial_{G} C_{n}$ and $\partial_{G} C_{n+1}$ for each $n \geq I$ and by piecing together the resulting paths we obtain a set of $\left|\partial_{G} C_{I}\right|$ edge-disjoint rays in $\omega$ that start in $N\left(C_{I}\right)$. Therefore, every cut separating $S^{\prime}:=N\left(C_{I}\right) \cup S$ from $\omega$ has cardinality at least (and also at most) $\left|\partial_{G} C_{I}\right|$. Thus, by (i) and Corollary $12, \omega$ has odd degree.

Let us give an outline of the idea of how the desired sequence $C_{1}, C_{2}, \ldots$ will be constructed. Since by assumption $G$ has an odd cut, we can start with a smallest such, and take one of the corresponding odd regions as $C_{1}$. It is not difficult to find a region $C_{2}$ that satisfies conditions (i) and (ii), as they hold for any one of the odd components of $C_{1}-N\left(G-C_{1}\right)$. Observe that such a component $C^{\prime}$ indeed exists, since $G$ is locally finite and all its vertices have even degree.

Now, choosing for $C_{2}$ an infinite odd region in $C_{1}-N\left(C_{1}\right)$ that has minimal odd cut ensures already half of condition (iii): then, for every odd region $D$ with $C_{1} \supseteq D \supseteq C_{2}$, clearly $|\partial D| \geq\left|\partial C_{1}\right|$. But what about the even regions sandwiched between $C_{1}$ and $C_{2}$ ? This is the main problem in our proof of Theorem 4.

To overcome this problem we first contract all infinite even regions $D \subseteq C_{1}$ with $|\partial D|<\left|\partial C_{1}\right|$. Only then we choose a region $C_{2}^{\prime}$ with minimal odd cut as above, which can be decontracted to a region $C_{2}$ of $G$ that satisfies condition (iii). When we contract the even infinite regions with small cut we need to be careful that in the obtained minor $G^{\prime}$ each vertex still has even degree. In order to guarantee this, the regions we contract have to be disjoint. This makes it impossible to contract each infinite region with too small cut, but by contracting a suitable selection of them we accomplish that all vanish.

Having found $C_{2}$, we proceed analogously with $C_{3}, C_{4}, \ldots$, which gives the desired sequence.

So let us now formally construct the sequence $C_{1}, C_{2}, \ldots$ satisfying (i)-(iii). For this, we need a further condition for $n \geq 1$. Let us call a region $C$ of a graph $H$ a $k$-region if $\left|\partial_{H} C\right|=k$.

(iv) for every $k$-region $D \subseteq C_{n}$ of $G$ with $k<\left|\partial_{G} C_{n}\right|$ there is an $\ell \in \mathbb{N}$ and even regions $K_{1}, \ldots, K_{\ell} \subseteq C_{n}$ such that $\left|\partial_{G} K_{i}\right| \leq k$ for all $i$, and $V(D) \subseteq \bigcup_{i=1}^{\ell} V\left(K_{i}\right)$. 
This condition, of course, is trivially satisfied if $k$ is even.

As $E(G) \notin \mathcal{C}(G)$, Theorem 7 ensures the existence of odd regions in $G$. Choose any odd region $C_{1}$ such that $\partial_{G} C_{1}$ has minimal cardinality. This choice satisfies (i) and (iv), and that is all we required for $n=1$.

Now, suppose the $C_{i}$ to be defined for $i \leq n$. In order to find a suitable $C_{n+1}$, we shall contract certain even $k$-regions $D$ of $G$ (contained in $C_{n}$ ) for which $k<\left|\partial_{G} C_{n}\right|$. In the resulting minor, which has only big cuts, we will choose a small odd cut, which in $G$ induces the desired region $C_{n+1}$. We should point out that whenever we contract an edge we keep parallel edges but delete all loops.

We will construct this minor in several steps. More precisely, for each even integer $m<\left|\partial_{G} C_{n}\right|$ we define a minor $G^{m}$ of $G=: G^{0}$, which will have the properties:

(a) $G^{m}$ is obtained from $G^{m-2}$ by contracting disjoint infinite $m$-regions $K$ of $G^{m-2}$ with $E(K) \subseteq E\left(C_{n}\right)$ for $m \geq 2$; and

(b) $\left|E(D) \cap E\left(G^{m}\right)\right|<\infty$ for every $k$-region $D$ of $G$ with $D \subseteq C_{n}$ and $k \leq m$.

Observe that, by (a) and as all vertices of $G$ are even, all vertices of $G^{m}$ have even degree too. In (a), we think of $K$ being contained in $C_{n}$. However, this is formally incorrect because $K$ lies in a minor of $G$ but $C_{n}$ is a region of $G$. Therefore, we use the slightly awkward requirement that $E(K) \subseteq E\left(C_{n}\right)$.

We claim that (b) together with (iv) implies for $m<\left|\partial_{G} C_{n}\right|-2$ :

(c) every $k$-region $D$ of $G^{m}$ with $E(D) \subseteq E\left(C_{n}\right)$ and $k \leq m+1$ is finite.

Note that, in a way, (c) may be viewed as a small improvement on (b). To prove (c), consider a $k$-region $D$ of $G^{m}$ with $E(D) \subseteq E\left(C_{n}\right)$ and $k \leq m+1$. By uncontracting, we obtain from $D$ a region $D^{\prime}$ of $G$ with $\partial_{G} D^{\prime}=\partial_{G^{m}} D$ and $E(D) \subseteq E\left(D^{\prime}\right)$. Since by assumption $m<\left|\partial_{G} C_{n}\right|-2$, we get that $k<\left|\partial_{G} C_{n}\right|$. Then (iv) implies that there is a finite set $\mathcal{K}$ of regions $K \subseteq C_{n}$ such that their union contains all vertices of $D^{\prime}$ (and thus also all but finitely many edges of $D^{\prime}$ ). Each $K \in \mathcal{K}$ is an $\ell$-region with even $\ell \leq k=m+1$. As $m+1$ is odd we get $\ell \leq m$, and hence by (b), that $E(K) \cap E\left(G^{m}\right)$ is finite. Thus $\left|E\left(D^{\prime}\right) \cap E\left(G^{m}\right)\right|<\infty$, and hence $D$ is finite. This establishes (c).

Let us now start defining the minors $G^{m}$. As $G$ is connected, $G^{0}=G$ obviously satisfies (b), which is all we required for $m=0$. So, assume $m \geq 2$, and $G^{i}$ to be constructed for all even $i<m$. We define a sequence $\left(L_{j}\right)_{j \in \mathbb{N}}$ of (not necessarily induced) subgraphs of $G^{m-2}$; by contracting the components of their union $L$ we obtain $G^{m}$.

Consider an enumeration $R_{1}, R_{2}, \ldots$ of all infinite $m$-regions of $G^{m-2}$ with $E\left(R_{i}\right) \subseteq E\left(C_{n}\right)$ (such an enumeration is possible since $E\left(G^{m-2}\right) \subseteq E(G)$ is countable). Put $L_{1}:=R_{1}$, and let for $j>1$,

$$
L_{j}:=L_{j-1} \cup R_{j} \text { if } \partial_{G^{m-2}} R_{j} \cap E\left(L_{j-1}\right)=\emptyset
$$

and $L_{j}:=L_{j-1}$ otherwise. Note that in the former case each component of $L_{j-1}$ is either contained in $R_{j}$ or disjoint from $R_{j}$. Thus, by induction on $j$, every component $K$ of $L_{j}$ is an infinite $m$-region of $G^{m-2}$.

Put $L:=\bigcup_{j \in \mathbb{N}} L_{j}$, and consider a component $K$ of $L$. Certainly, $K$ is an infinite induced subgraph in $G^{m-2}$ with $E(K) \subseteq E\left(C_{n}\right)$. We claim that 
$k:=\left|\partial_{G^{m-2}} K\right|=m$. Clearly, $k \leq m$ as otherwise there would already be a component $K^{\prime} \subseteq K$ of some $L_{j}$ with $\left|\partial_{G^{m-2}} K^{\prime}\right|>m$, which is impossible. On the other hand, $k \geq m$, by (c) for $m-2$; thus $k=m$, as desired. We now obtain $G^{m}$ from $G^{m-2}$ by contracting the components of $L$ to one vertex each (keeping multiple edges but deleting loops). Obviously, $G^{m}$ satisfies (a).

At first glance, it might look simple to prove (b): since when defining $G^{m}$ we contract (most of the) infinite $m$-regions, surely there should not be any infinite $k$-regions with small $k$ left in $G^{m}$. While this is true and not difficult to show, it is not enough for (b). The problem here is that (b) is a statement about regions of $G$ rather than of $G^{m}$. For instance, while contracting $G$ to $G^{m}$, we might contract edges of $\partial_{G} D$ for some region $D$ of $G$. Then, $D$ will not correspond to a region $D^{\prime}$ of $G^{m}$, and we cannot (directly) use our knowledge on regions in $G^{m}$ to deduce anything about $D$.

To circumvent this problem, before we show the validity of (b) we first present a tool that will allow us to transform a region $D$ of $G$ into an induced subgraph $D^{\prime}$ for which $\partial_{G} D^{\prime} \subseteq G^{m}$ and which does not differ from $D$ "too much". More precisely, we prove that for all even $i<m<\left|\partial_{G} C_{n}\right|$ it holds that:

(*) for every region $D \subseteq C_{n}$ of $G$ with $\partial_{G} D \subseteq E\left(G^{i}\right)$ there is a (possibly empty) induced subgraph $D^{\prime} \subseteq C_{n}$ that satisfies

(I) there are finitely many regions $K_{1}, \ldots, K_{\ell}$ of $G$ each of which contracts to a vertex of degree $\leq i+2$ in $G^{i+2}$ such that $V(D) \backslash V\left(D^{\prime}\right) \subseteq$ $\bigcup_{j=1}^{\ell} V\left(K_{j}\right) ;$

(II) if there is a region $C$ of $G$ with $D \subseteq C$ and $\partial_{G} C \subseteq E\left(G^{i+2}\right)$, then also $D^{\prime} \subseteq C$;

(III) $\left|\partial_{G} D^{\prime}\right| \leq\left|\partial_{G} D\right|$; and

(IV) $\partial_{G} D^{\prime} \subseteq E\left(G^{i+2}\right)$.

Observe that $D^{\prime}$ has at most $\left|\partial_{G} D^{\prime}\right|$ components (since $G$ is connected). Each of these is a region of $G$ with properties (II)-(IV).

Let us now prove $(*)$. Given $D$ as above, choose an induced subgraph $\tilde{D} \subseteq$ $C_{n}$ such that $\left|\partial_{G} \tilde{D} \backslash E\left(G^{i+2}\right)\right|$ is minimal among all induced subgraphs that satisfy (I), (II), (III) and $\partial_{G} \tilde{D} \subseteq E\left(G^{i}\right)$ (which is possible as $D$ itself has these four properties). If $\left|\partial_{G} \tilde{D} \backslash E\left(G^{i+2}\right)\right|=0$, we may put $D^{\prime}:=\tilde{D}$, so suppose otherwise. Then, by (a), there is an $(i+2)$-region $K$ of $G^{i}$ with $E(K) \subseteq E\left(C_{n}\right)$, which is contracted to a vertex in $G^{i+2}$ and for which holds that $\partial_{G} \tilde{D} \cap E(K) \neq$ $\emptyset$. Denote by $\tilde{D}^{i}$ the image of $\tilde{D}$ in $G^{i}$, i.e. the induced subgraph of $G^{i}$ with $\partial_{G^{i}} \tilde{D}^{i}=\partial_{G} \tilde{D}$ and $E(\tilde{D}) \cap E\left(G^{i}\right)=E\left(\tilde{D}^{i}\right)$.

Suppose that one of $\left|E_{G^{i}}\left(K \cap \tilde{D}^{i}, \tilde{D}^{i} \backslash K\right)\right|,\left|E_{G^{i}}\left(K \backslash \tilde{D}^{i}, G^{i}-\left(\tilde{D}^{i} \cup K\right)\right)\right|$ is smaller than or equal to $\left|E_{G^{i}}\left(K \cap \tilde{D}^{i}, K \backslash \tilde{D}^{i}\right)\right|$. Then, putting either $\hat{D}^{i}=\tilde{D}^{i} \backslash K$ or $\hat{D}^{i}=G^{i}\left[\tilde{D}^{i} \cup K\right]$ we get

$$
\left|\partial_{G^{i}} \hat{D}^{i}\right| \leq\left|\partial_{G^{i}} \tilde{D}^{i}\right|=\left|\partial_{G} \tilde{D}\right| .
$$

Observe that $\partial_{G^{i}} \hat{D}^{i} \cap E(K)=\emptyset$, and denote by $\hat{D}$ the induced subgraph of $G$ that we obtain from $\hat{D}^{i}$ by uncontracting. Since the edges of $K$ are contracted in $G^{i+2}$ and since, by construction, no new edges outside $E\left(G^{i+2}\right)$ are introduced in $\partial_{G} \hat{D}=\partial_{G^{i}} \hat{D}^{i}$ it follows that $\partial_{G} \hat{D}$ has fewer edges in $E(G) \backslash E\left(G^{i+2}\right)$ than $\partial_{G} \tilde{D}$. We claim that this contradicts the minimal choice of $\tilde{D}$. Indeed, $\partial_{G} \hat{D} \subseteq E\left(G^{i}\right)$, 


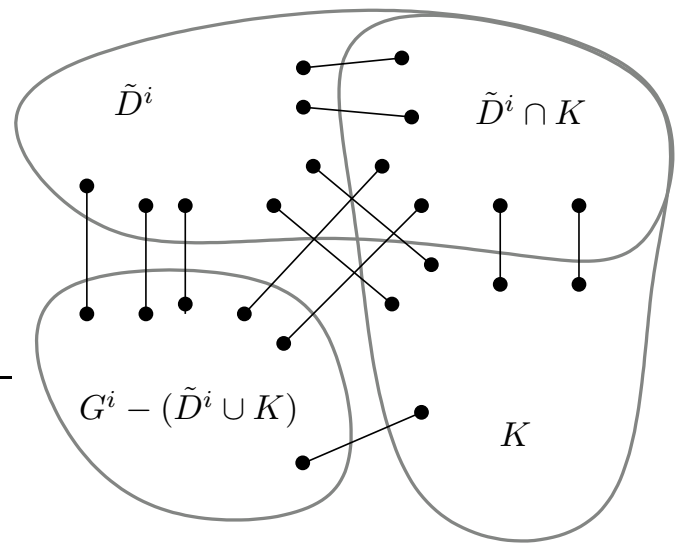

Figure 4: $\tilde{D}^{i}$ and $K$ in $G^{i}$

and also (I) and (III) hold for $\hat{D}$ : the latter by (2), and for the former observe that each $K_{i}$ either still contracts to a vertex of degree $\leq i$ in $G^{i+2}$ or, failing that, is contained in a region that contracts to a vertex of degree $i+2$ (this might happen if the vertex $v_{K_{i}}$ to which $K_{i}$ is contracted in $G^{i}$ lies in a region of $G^{i}$ that gets contracted when going from $G^{i}$ to $G^{i+2}$ ). Adding $K$ to these regions, we obtain the $K_{i}$ as desired for (I).

To see that $\hat{D}$ satisfies (II), consider a region $C \supseteq D$ with $\partial_{G} C \subseteq E\left(G^{i+2}\right)$. Observe that $\tilde{D} \subseteq C$ because $\tilde{D}$ satisfies (II). Now, since $\partial_{G} C \cap E(K)=\emptyset$, either $E(K) \subseteq E(C)$ or $E(K) \subseteq E(G-C)$ because $K$ is connected. The latter case is impossible, as $\partial_{G} \tilde{D} \cap E(K) \neq \emptyset$. Hence, $E(K) \subseteq E(C)$, and thus, as $\hat{D}^{i} \subseteq G^{i}\left[\tilde{D}^{i} \cup K\right]$, we get $\hat{D} \subseteq C$, as desired. Note also that $\hat{D} \subseteq C_{n}$, as $\partial_{G} C_{n} \subseteq E\left(G^{i+2}\right)$ by (a).

We may therefore assume that

$\left|E_{G^{i}}\left(K \cap \tilde{D}^{i}, K \backslash \tilde{D}^{i}\right)\right|<\left|E_{G^{i}}\left(K \cap \tilde{D}^{i}, \tilde{D}^{i} \backslash K\right)\right|,\left|E_{G^{i}}\left(K \backslash \tilde{D}^{i}, G^{i}-\left(\tilde{D}^{i} \cup K\right)\right)\right|$, and thus $\left|\partial_{G^{i}}\left(K \cap \tilde{D}^{i}\right)\right|,\left|\partial_{G^{i}}\left(K \backslash \tilde{D}^{i}\right)\right|<\left|\partial_{G^{i}} K\right|$. As $K$ is infinite and as $K \cap \tilde{D}^{i}$ and $K \backslash \tilde{D}^{i}$ have only finitely many components (since $G$ is connected), one of these components, say $K^{\prime}$, is infinite. Now, $K^{\prime}$ is a region of $G^{i}$ with $\partial_{G^{i}} K^{\prime} \subseteq$ $\partial_{G^{i}}\left(K \cap \tilde{D}^{i}\right)$ or $\partial_{G^{i}} K^{\prime} \subseteq \partial_{G^{i}}\left(K \backslash \tilde{D}^{i}\right)$. In both cases, $\left|\partial_{G^{i}} K^{\prime}\right|<\left|\partial_{G^{i}} K\right|=$ $i+2$. Because $E\left(K^{\prime}\right) \subseteq E(K) \subseteq E\left(C_{n}\right)$ and $i \leq m-2<\left|\partial_{G} C_{n}\right|-2$, this contradicts (c). We have thus shown $(*)$.

Let us prove that $G^{m}$ also satisfies (b). For this, consider a region $D \subseteq C_{n}$ of $G$ with $\left|\partial_{G} D\right| \leq m$, and suppose that $E(D) \cap E\left(G^{m}\right)$ is infinite. Assume $D$ to be chosen among all such regions such that $i$ is maximal with $\partial_{G} D \subseteq E\left(G^{i}\right)$. Now, if $i<m$, then $(*)$ yields a subgraph $D^{\prime}$. By (I), all but finitely many of the edges in $E(D) \cap E\left(G^{m}\right)$ lie in $E\left(D^{\prime}\right)$. Since $D^{\prime}$ has only finitely many components, there is one, $C$ say, such that $E(C) \cap E\left(G^{m}\right)$ is infinite. Since, by (III), $\left|\partial_{G} C\right| \leq\left|\partial_{G} D\right|$, and since, by (IV), $\partial_{G} C \subseteq E\left(G^{i+2}\right)$, we obtain a contradiction to the choice of $D$. Thus, we may assume that $i=m$, i.e. $\partial_{G} D \subseteq$ $E\left(G^{m}\right)$.

Therefore, by performing the according contractions we obtain from $D$ an infinite region $\tilde{D}$ of $G^{m-2}$ such that $\partial_{G^{m-2}} \tilde{D}=\partial_{G} D$ and $E(\tilde{D}) \subseteq E\left(C_{n}\right)$. Be- 
cause of (c) for $m-2$ and because of $\left|\partial_{G} D\right| \leq m$, we get $\left|\partial_{G^{m-2}} \tilde{D}\right|=m$. Hence, the region $\tilde{D}$ appears in the enumeration $R_{1}, R_{2}, \ldots$ used in the construction of $G^{m}$, i.e. there is a $j$ with $\tilde{D}=R_{j}$. Suppose that $E\left(R_{j}\right) \nsubseteq E\left(L_{j}\right)$. Then, by (1), $\partial_{G^{m-2}} R_{j} \cap E\left(L_{j-1}\right) \neq \emptyset$, but each edge in $L_{j-1}$ will be contracted in the construction of $G^{m}$, contradicting $\partial_{G^{m-2}} R_{j} \subseteq E\left(G^{m}\right)$. Therefore, it holds that $E\left(R_{j}\right) \subseteq E\left(L_{j}\right) \subseteq E(L)$. Thus, in $G^{m}$ all edges of $\tilde{D}=R_{j}$ are contracted, a contradiction to $\left|E(D) \cap E\left(G^{m}\right)\right|=\infty$. This establishes (b).

Having constructed $G^{m}$ for all $m \leq M:=\left|\partial_{G} C_{n}\right|-1$, we finally find the region $C_{n+1}$. Observe that, by (a), $\partial_{G} C_{n}$ is a cut of $G^{M}$, and that the cut $F$ of $G^{M}$ that consists of those edges in $E\left(G^{M}\right) \cap E\left(C_{n}\right)$ that in $G^{M}$ are adjacent to $\partial_{G} C_{n}$ has odd cardinality (because by (a), all vertices of $G^{M}$ are even). Thus, since $F$ is also a cut of $G$, there exists a region $C$ of $G$ with $\partial_{G} C \subseteq E\left(G^{M}\right)$ that satisfies (i) and (ii) for $n+1$.

We claim that

any region $C$ of $G$ that for $n+1$ satisfies (i), (ii), and $\partial_{G} C \subseteq E\left(G^{M}\right)$, also satisfies (iii).

First, observe that $E(C) \cap E\left(G^{M}\right)$ is infinite. Indeed, by $\partial_{G} C \subseteq E\left(G^{M}\right)$ and by (i), $C$ contracts to an odd region in $G^{M}$. An odd region in a graph in which all vertices are even is always infinite (and all vertices of $G^{M}$ are indeed even, by $(\mathrm{a}))$.

Now, consider a $k$-region $D$ of $G$ with $C_{n} \supseteq D \supseteq C$. From (b) for $m=M$ it follows that $k \geq M+1=\left|\partial_{G} C_{n}\right|$, as desired for (iii). This establishes (3).

Choose $C_{n+1}$ such that $\partial_{G} C_{n+1}$ has minimal (odd) cardinality among all regions satisfying (i), (ii) and (iii) for $n+1$.

To see (iv) for $n+1$, consider a $k$-region $D \subseteq C_{n+1}$ with $k<\left|\partial_{G} C_{n+1}\right|$. If $k \leq M$, then we can apply (iv) for $n$, so suppose $k>M$. Furthermore, we may assume that $k$ is odd, as otherwise we can choose $\ell:=1$ and $K_{1}:=D$. Hence $D$ satisfies (i) and (ii) for $n+1$, and we should have chosen $D$ as $C_{n+1}$, if not (iii) and thus, by (3), also $\partial_{G} D \subseteq E\left(G^{M}\right)$ fails for $D$. Take the smallest $i_{D} \leq M$ such that $\partial_{G} D \nsubseteq E\left(G^{i_{D}}\right)$. Then, repeated use of $(*)$ for $i=i_{D}-2, i_{D}, \ldots, M-2$, where we apply $(*)$ in each step to every component of the subgraph $D^{\prime}$ obtained in the previous step, yields a subgraph $D^{*}$ of $C_{n}$ such that each of its components $K_{1}, K_{2}, \ldots, K_{\ell}$ has properties (II)-(IV) for $m=M$. In particular, (II) implies for $1 \leq i \leq \ell$ that $K_{i} \subseteq C_{n+1}$. Let $\left\{K_{\ell+1}, \ldots, K_{L}\right\}$ be the set of all regions that arose as one of the $K_{i}$ in one of the applications of $(*)$. Then, by (I),

$$
V(D) \subseteq V\left(D^{*}\right) \cup \bigcup_{i=\ell+1}^{L} V\left(K_{i}\right)=\bigcup_{i=1}^{L} V\left(K_{i}\right) .
$$

By (III), $\left|\partial_{G} K_{i}\right| \leq k$ for $i=1, \ldots, \ell$. Now, if there is an $j \in\{1, \ldots, \ell\}$ such that $\left|\partial_{G} K_{j}\right|$ is odd, then $K_{j} \subseteq C_{n+1}$ satisfies (i), (ii), and, by (IV) and (3), also (iii) for $n+1$, contradicting the choice of $C_{n+1}$. So, $\left|\partial_{G} K_{i}\right|$ is even and $\leq k$ for $i=1, \ldots, L$ (for $i>\ell$ this follows from (I) and $k>M$ ). As $\partial_{G} C_{n+1} \subseteq E\left(G^{M}\right)$, and thus $\partial_{G} C_{n+1} \cap \bigcup_{i=\ell+1}^{L} E\left(K_{i}\right)=\emptyset$, and as $K_{1}, \ldots, K_{\ell} \subseteq C_{n+1}$, each of the $K_{1}, \ldots, K_{L}$ either lies completely in $C_{n+1}$ or is disjoint from it. Together with $D \subseteq C_{n+1}$ this implies that $V(D) \subseteq \bigcup_{K \in \mathcal{K}} V(K)$ for $\mathcal{K}:=\left\{K_{i}: K_{i} \subseteq C_{n+1}\right.$ and $1 \leq i \leq L\}$, which proves (iv) for $n+1$. This completes the proof of the theorem. 


\section{Properties of the end degree}

As an indication that the end degree indeed behaves as expected of a degree, we extend three basic properties of the vertex degree in finite graphs to end degrees in locally finite graphs. At the end of this section, however, we present two examples where end degrees differ from vertex degrees.

The number of odd vertices in a finite graph is always even. We prove the following easy analogue.

Proposition 15. Let $G$ be a locally finite graph. Then the number of odd vertices and ends in $G$ is even or infinite.

Proof. Suppose that the set $\mathcal{O}$ of odd vertices and ends has odd cardinality. Observe that there is a finite set $S \subseteq V(G)$ that contains all vertices of $\mathcal{O}$ and separates the ends in $\mathcal{O}$ pairwisely. By Corollary 12, there is for each end $\omega \in \mathcal{O}$ an odd region $A_{\omega} \subseteq G-S$ with $\omega \in \overline{A_{\omega}}$. Observe that the $A_{\omega}$ are pairwise disjoint. So, contracting each $A_{\omega}$ to a vertex $a_{\omega}$ we arrive at a graph $G^{\prime}$ that has an odd number of odd vertices. Moreover, all ends of $G^{\prime}$ have even degree. Indeed, each end $\omega$ of $G^{\prime}$ corresponds to an even end in $G$, and we easily find a region $D \subseteq G \cap G^{\prime}$ to which $\omega$ belongs. Since parity is a local property $\omega$ is thus not only even in $G$ but also in $G^{\prime}$. Now, consider two copies of $G^{\prime}$ and add all edges $v v^{\prime}$, where $v$ is an odd vertex of $G^{\prime}$ and $v^{\prime}$ its copy. The resulting graph has an odd cut, but no odd vertices or ends, a contradiction to Theorems 4 and 7 .

Dirac [7] observed that if a finite graph has minimum vertex degree $k \geq 2$ then it contains a circuit of length $k+1$. This becomes false for infinite graphs: an easy counterexample is the $k$-regular infinite tree. But the tree ceases to be a counterexample if a minimum degree is also imposed on the ends, and indeed, then Dirac's result extends to locally finite graphs:

Theorem 16. Let $G$ be a locally finite graph, and let $H \subseteq G$ be a subgraph so that every vertex and every end $x \in \bar{H}$ has degree at least $k \geq 2$ in $H$. Then there is a circuit $C \subseteq E(H)$ of $G$ with length $\geq k+1$.

First, note that the theorem is best possible, even for infinite graphs. Indeed, consider disjoint copies $G_{1}, G_{2}, \ldots$ of $K^{k+1}$. Identify a vertex in $G_{1}$ with a vertex in $G_{2}$. Then identify a different vertex in $G_{2}$ with a vertex of $G_{3}$ and so on. In the resulting graph the minimum vertex degree is $k$, and it is easy to see that the single end has degree $k$ too, but there is no circuit of length greater than $k+1$. See Figure 5 for an example with $k=2$.

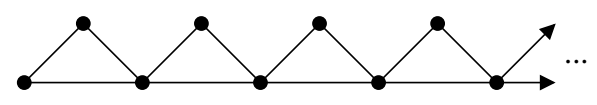

Figure 5: Theorem 16 is best possible for $k=2$

Next, let us remark that the long circuit provided by the theorem may be infinite, and indeed the result becomes false if we require finite circuits. To see this, consider a $k$-regular tree $H$ with root $r$. Let $G$ be the graph obtained by adding an edge between any two vertices which the same distance to $r$. Then 
$G$ has a single end, which has infinite degree in $H$, but $H$ does not contain any finite circuits.

For the proof, we need a simple lemma that shows how we can construct a topological path by piecing together infinitely many arcs.

Lemma 17. Let $G$ be a locally finite graph, and let for $n \in \mathbb{N}, \phi_{n}:[0,1] \rightarrow|G|$ be a homeomorphism such that if $A_{n}:=\phi_{n}([0,1])$ it holds that:

(i) $A_{n} \cap A_{m} \subseteq V(G) \cup \Omega(G)$ for $n \neq m$; and

(ii) $\phi_{n}(1)=\phi_{n+1}(0)$ for all $n$.

Then there is an $x \in|G|$ such that $\bigcup_{n=1}^{\infty} A_{n} \cup\{x\}$ is a topological path from $\phi_{1}(0)$ to $x$.

Proof. Instead of the $\phi_{n}$ let us consider compositions with suitable homeomorphisms $\phi_{n}^{\prime}:\left[1-2^{-(n-1)}, 1-2^{-n}\right] \rightarrow A_{n}$. Together the $\phi_{n}^{\prime}$ define, by (ii), a continuous function $\phi^{\prime}:[0,1) \rightarrow|G|$. As $|G|$ is compact, the sequence $\phi_{1}(0)=\phi^{\prime}(1 / 2), \phi_{2}(0)=\phi^{\prime}(3 / 4), \ldots$ has an accumulation point $x$. We claim that $\phi:[0,1] \rightarrow|G|$ defined by $\phi(s):=\phi^{\prime}(s)$ for $s \in[0,1)$ and by $\phi(1):=x$ is continuous.

Let a neighbourhood $V$ of $x$ be given, and note that because of (i) and (ii), none of the $\phi_{n}(0)$ is an inner point of an edge, and thus $x$ is an end. Then there is a basic open neighbourhood $\hat{C}(S, x) \subseteq V$ that contains all but finitely many of the $\phi_{n}(0)$. By (i), only finitely many of the $A_{n}$ meet the finite cut $\partial C(S, x)$. So, there is an $N$ such that $A_{n} \subseteq \hat{C}(S, x)$ for $n \geq N$. Consequently, $\phi^{-1}(V)$ contains the open set $\left(1-2^{-N}, 1\right]$, and thus is a neighbourhood of 1 in $[0,1]$.

Proof of Theorem 16. First, observe that if $H$ has an infinite block then $H$ contains two disjoint rays that are equivalent in $H$ (and thus also in $G$ ). By linking these by a path in $H$ we obtain a double ray whose edge set is an infinite circuit of $G$.

Therefore, we may assume that every block of $H$ is finite. Next, suppose that there is a block $B$ of $H$ that contains at most one vertex $v$ with $d_{B}(v)<k$. Pick a longest path in $B$. One of the endvertices has at least $k$ neighbours on that path, and hence there is a finite circuit of length $\geq k+1$ in $B$.

So, every block $B$ of $H$ is finite and contains at least two vertices of $H$ with degree $<k$ in $B$, which then are cutvertices of $H$. Now, replace every block $B$ of $H$ by a (not necessarily spanning) tree $T \subseteq B$ whose leaves are exactly the cutvertices of $H$ incident with $B$. Then every vertex of the resulting forest $H^{\prime} \subseteq H$ has degree $\geq 2$ as every block contains at least two cutvertices.

Assume that $E\left(H^{\prime}\right)$ does not contain infinite circuits, and let $v_{1}, v_{2}, \ldots$ be an enumeration of $V\left(H^{\prime}\right)$. We will inductively construct for $n \in \mathbb{N}$ homeomorphisms $\phi_{n}:[0,1] \rightarrow \overline{H^{\prime}} \subseteq|G|$. Choosing $b_{0}$ as any vertex in $H^{\prime}$ and putting $A_{n}:=\phi_{n}([0,1])$, we require that for $n \geq 1$ both $a_{n}:=\phi_{n}(0)$ and $b_{n}:=\phi_{n}(1)$ are vertices, and satisfy:

(i) $a_{n}=b_{n-1}$ for $n \geq 2$;

(ii) $A_{m} \cap A_{n}=\emptyset$ for $1 \leq m \leq n-2$ and $A_{n-1} \cap A_{n}=\left\{b_{n-1}\right\}$;

(iii) there is a cutvertex $v$ incident with two blocks $B, B^{\prime}$ of $H$ such that $d_{B}(v)<k$ and such that $A_{n}$ contains two edges incident with $v$, one 
in $E(B)$ and the other in $E\left(B^{\prime}\right)$ (let us call any arc with that property deficient); and

(iv) if there is a topological path in $\overline{H^{\prime}}$ from $b_{n-1}$ to $v_{n}$ that is edge-disjoint from $B_{n-1}:=\bigcup_{i=1}^{n-1} A_{i}$, then $v_{n} \in A_{n}$.

Note that for $n \geq 1, B_{n}$ is a topological path.

In order to construct $\phi_{n}$, assume $\phi_{1}, \ldots, \phi_{n-1}$ to be defined already. First, suppose there is a topological path as required by (iv). By Lemma 6, either $b_{n-1}$ and $v_{n}$ are the endpoints of an arc $A$ that is edge-disjoint from $B_{n-1}$, or $b_{n-1}=v_{n}$, in which case we put $A:=\left\{v_{n}\right\}$. We claim that $A \cap B_{n-1}=\left\{b_{n-1}\right\}$. Indeed, otherwise let $v$ be the vertex with $b_{n-1} v \subseteq A$. Then $A \cup B_{n-1}$ contains a topological path from $v$ to $b_{n-1}$ that avoids all inner points of $b_{n-1} v$, and hence, by Lemma 6 , also a $b_{n-1}-v$ arc $A^{\prime}$. Thus, $A^{\prime} \cup b_{n-1} v \subseteq A \cup B_{n-1} \subseteq \overline{H^{\prime}}$ is a circle, contradicting our assumption.

We now lengthen $A$ so that it also satisfies (iii). Because every vertex has degree $\geq 2$ in $H^{\prime}$, and because $\overline{H^{\prime}}$ does not contain any circles, $v_{n}$ has a neighbour in $H^{\prime} \backslash A \cup B_{n-1}$. Continuing in this way, we obtain a $v_{n}-B$ path in $H^{\prime}$ that meets $A \cup B_{n-1}$ only in $v_{n}$, where $B$ is a block of $H$ which is adjacent to the block that contains $v_{n}$. As $B \cap \overline{H^{\prime}}$ is connected and as $\overline{H^{\prime}}$ does not contain any circles, $B$ is disjoint from $A \cup B_{n-1}$. So, since $B$ has a cutvertex $b$ with $d_{B}(b)<k$, there is a deficient path $P \subseteq H^{\prime}$ that starts in $v_{n}$ and is otherwise disjoint from $A \cup B_{n-1}$. Thus, we easily find a homeomorphism $\phi_{n}:[0,1] \rightarrow A \cup P$ which satisfies (i)-(iv).

So suppose there is no topological path as in (iv). Again we find a deficient path $P \subseteq H^{\prime}$ starting in $b_{n-1}$ which is disjoint from $B_{n-1} \backslash\left\{b_{n-1}\right\}$, and the respective homeomorphism $\phi_{n}:[0,1] \rightarrow P$ has properties (i)-(iv).

This process yields a set of arcs $A_{n}$, to which we apply Lemma 17 . We obtain an $x \in|G|$, which is necessarily an end, such that $A^{*}:=\bigcup_{n=1}^{\infty} A_{n} \cup\{x\}$ is a topological path from $b_{0}$ to $x$.

The end $x$ has degree $k$ in $H$, and hence there are $k$ edge-disjoint arcs $R_{1}, \ldots, R_{k} \subseteq \bar{H}$ that start in $x$. Each of the $R_{i}$ meets $A^{*} \backslash\{x\}$ in every neighbourhood of $x$. Indeed, suppose there is a neighbourhood $U$ of $x$ and an index $j$ such that $R_{j} \cap U$ is disjoint from $A^{*} \backslash\{x\}$. Since $R_{j}$ is continuous, there is a subarc of $R_{j}$ which starts in $x$ and is completely contained in $U$. Pick a vertex $v_{m}$ on this subarc, and denote by $R$ the subarc of $R_{j}$ between $x$ and $v_{m}$. Then $\bigcup_{n=m-1}^{\infty} A_{n} \cup R$ clearly is a topological path from $b_{m-1}$ to $v_{m}$ which is edge-disjoint from $B_{m-1}$, a contradiction to (iv) as $v_{m} \notin A^{*} \supseteq A_{m}$.

Let $\phi:[0,1] \rightarrow A^{*}$ be a continuous function with range $A^{*}$ and $\phi(1)=x$. Choose an $s \in[0,1)$ such that each of the $R_{i}$ hits $A^{*}$ in a $\phi\left(r_{i}\right)$ with $r_{i}<s$. Because of (iii), we may assume that $v:=\phi(s)$ is a cutvertex incident with two blocks $B, B^{\prime}$ of $H$ such that $d_{B}(v)<k$ and such that $A^{*}$ contains two edges incident with $v$, one in $E(B)$ and the other in $E\left(B^{\prime}\right)$. Not all of the $k$ arcs $R_{i}$ can go through the cut $F:=E_{H}(v, B-v)$ of $H$, which has cardinality $d_{B}(v)<k$; so assume $R_{j}$ does not contain any edge of $F$. Let $u w$ be the (unique) edge in $E(A) \cap F$, and assume $\phi^{-1}(u) \leq \phi^{-1}(w)$ (note that either $u=v$ or $w=v$ ). Then $\left(A \cup R_{j}\right) \backslash u w \cup\{u, w\}$ contains a topological path from $w$ to $u$ (simply run from $w$ to $x$ along $A$, then from $x$ to $\phi\left(r_{j}\right)$ along $R_{j}$ and finally from $\phi\left(r_{j}\right)$ to $u$ along $A)$. Therefore, there is also an arc $R \subseteq\left(A \cup R_{j}\right) \backslash u w \cup\{u, w\}$ with endpoints $u$ and $w$, by Lemma 6 . Consequently, $R \cup u w \subseteq \bar{H}$ is a circle. Since 
$E(R)$ is disjoint from $F$ and every $B-\left(B^{\prime}-v\right)$ path in $H$ has to go through $F$, $|E(R)|$ is infinite. Thus, $E(R \cup u w) \subseteq E(H)$ is an infinite circuit, as desired.

In a finite graph the cycles are exactly the connected 2-regular subgraphs. The same is true for locally finite graphs.

Proposition 3. Let $C$ be a subgraph of a locally finite graph $G$. Then $\bar{C}$ is a circle if and only if $\bar{C}$ is topologically connected and every vertex or end $x$ of $G$ with $x \in \bar{C}$ has degree two in $C$.

We deduce Proposition 3 from Theorem 16. However, there is also a direct proof $^{1}$ of the proposition, which is not much more complicated.

Proof of Proposition 3. If $\bar{C}$ is a circle, then it is clearly topologically connected and every vertex and every end $x \in \bar{C}$ has degree two in $C$.

For the converse direction, Theorem 16 implies that there is a circle $D \subseteq \bar{C}$. Suppose there exists a point $z \in \bar{C} \backslash D$. Theorem 5 yields an arc $A \subseteq \bar{C}$ that starts at $z$ and ends in $D$. As both $A$ and $D$ are closed, $A$ has a first point in $D$, i.e. a point $x$ such that the subarc $A^{\prime}$ of $A$ between $z$ and $x$ meets $D$ only in $x$. Thus, there are three edge-disjoint arcs in $C$ with common endpoint $x$, two in $D$ and the arc $A^{\prime}$. So, $x$ is either a vertex or an end and has degree at least 3 in $C$, a contradiction. Thus, $\bar{C}=D$.

Let us now turn to two areas in which end degrees differ in their behaviour from vertex degrees in finite graphs.

For a subgraph $H$ of a graph $G$, deleting $E(H)$ reduces the degree of a vertex $v \in V(G)$ by its degree in $H$, i.e. $d_{G}(v)=d_{H}(v)+d_{G-E(H)}(v)$. Although for an end $\omega$ it clearly holds that $d_{G}(\omega) \geq d_{H}(\omega)+d_{G-E(H)}(\omega)$, equality is in general not ensured. Consider the $4 \times \infty$-grid, which has a single end. As depicted in Figure 6 , the removal of (the edge set of) a ray $R$ leads to a decrease of the end degree from 4 to any of 3,2,1 or 0 , depending on how $R$ is chosen. Similarly, deleting a circuit can lead to an odd decrease in the degree.
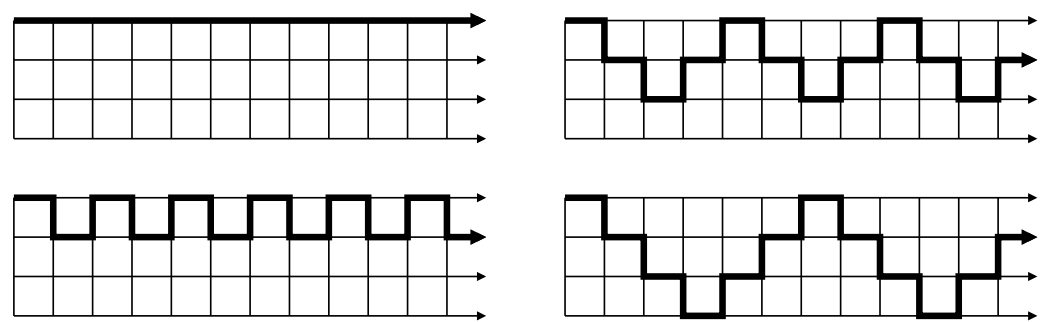

Figure 6: Removal of a ray lets the end degree decrease by 1 or more

The second area where the end degree differs in its behaviour concerns extremal results. A classical theorem of Mader [11], for instance, states that high

\footnotetext{
${ }^{1}$ Here is a sketch of the backward direction: Choose an edge $x y \in C$, and pick a maximal arc $A$ in $\bar{C}-x y$ that starts in $x$, which exists by Zorn's lemma. The endpoint $z$ of this arc has degree 2 in $\bar{C}$. Thus, if $z \neq y$, then $A$ meets both $z$-arcs in $\bar{C}$. But this is impossible as every vertex and every end in $\bar{C}$ has degree 2 .
} 
average degree forces a finite graph to contain a large complete minor. This, however, fails for locally finite graphs even if every end has high degree. Figure 7

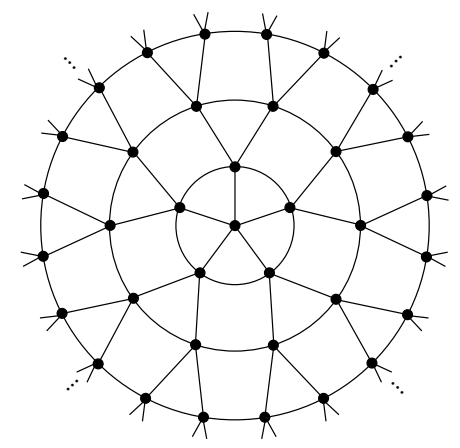

Figure 7: High degree in all vertices and in the single end but planar

indicates how for every $k \geq 5$ a planar $k$-regular graph with a single end of infinite degree can be constructed. Being planar, such a graph can never contain even a $K^{5}$ as a minor.

\section{Weakly even ends}

Finally, let us briefly discuss an alternative degree concept, which arises from the observation that (ii) of Lemma 8 is equivalent to:

(iii) for every finite $S \subseteq V(G)$ there is a finite set $S^{\prime} \supseteq S$ of vertices such that the maximal number of edge-disjoint $\omega$-rays starting in $S^{\prime}$ is even.

Lemma 8 (ii) was our main motivation for our definition of an even end. In the same vein, (iii) leads to the following alternative definition of parity, which differs only in that the quantifiers are exchanged:

Definition 18. Let $H$ be a subgraph of a locally finite graph $G$. Call $\omega$ weakly even in $H$ if for every finite $S \subseteq V(G)$ there is a finite set $S^{\prime} \supseteq S$ of vertices such that the maximal number of edge-disjoint $\omega$-arcs in $\bar{H}$ starting in $S^{\prime}$ is even. Otherwise, $\omega$ is strongly odd in $H$.

Observe that an even end is weakly even, and that a strongly odd end is odd. For ends of finite degree the two parity concepts are equivalent; this can be seen in a similar way as the equivalence of (ii) and (iii). For ends of infinite degree, however, this need not be true: consider a ray $v_{1} v_{2} \ldots$, and replace each edge $v_{i} v_{i+1}$ by $i$ (subdivided) parallel edges. The obtained graph has a single end, which is both odd and weakly even.

This construction only works because there are odd vertices present. But could an odd end exist in a graph that has all vertices even and all ends weakly even? Or, on the contrary:

Problem 19. Does Theorem 4 remain true if we substitute "even ends" by "weakly even ends"? 
We have been unable to settle the problem. However, the following proposition answers both this question and Conjecture 14 positively for locally finite graphs with only countably many ends. It should be noted that its proof uses Corollary 12 in its full generality, the proof of which we did not provide for the sake of brevity.

Proposition 20. Let $G$ be a locally finite graph with only countably many ends, and let $H$ a subgraph. Then $E(H) \in \mathcal{C}(G)$ if and only if every vertex has even degree in $H$ and if every end has weakly even degree in $H$.

Proof. The forward direction follows immediately from Theorem 7 and Corollary 12. For the backward direction, suppose $E(H) \notin \mathcal{C}(G)$, which by Theorem 7 means that $G$ has a finite cut $F$ with $|F \cap E(H)|$ odd. Let $\omega_{1}, \omega_{2}, \ldots$ be an enumeration of $\Omega(G)$. We successively define a sequence $\mathcal{A}_{0} \subseteq \mathcal{A}_{1} \subseteq \ldots$ of finite sets of disjoint regions $A \subseteq G-F$ of $G$ with $|\partial A \cap E(H)|$ even and such that for each $\omega_{i}$ with $i \leq n$ there is an $A \in \mathcal{A}_{n}$ with $\omega_{i} \in \bar{A}$. Put $\mathcal{A}_{0}:=\emptyset$. In order to define the set $\mathcal{A}_{n}$ first check whether there is an $A \in \mathcal{A}_{n-1}$ such that $\omega_{n} \in \bar{A}$, in which case we put $\mathcal{A}_{n}:=\mathcal{A}_{n-1}$. Otherwise consider the (finite) set $S$ of all neighbours of each $A \in \mathcal{A}_{n-1}$ and of the endvertices of the edges in $F$. As $\omega_{n}$ is weakly even, Lemma 10 yields a region $B \subseteq G-F$ with $\omega_{n} \in \bar{B}$ and $A \cap B=\emptyset$ for all $A \in \mathcal{A}_{n-1}$. Put $\mathcal{A}_{n}:=\mathcal{A}_{n-1} \cup\{B\}$. Finally, contracting all the disjoint regions $A \in \bigcup_{n=1}^{\infty} \mathcal{A}_{n}$ to a vertex each yields a finite graph with all vertex degrees even in $H$ that has a cut $F$ with $|F \cap E(H)|$ odd, a contradiction.

\section{Acknowledgement}

We would like to thank the anonymous referees for pointing out a number of improvements, one of these being the short direct proof of Proposition 3.

\section{References}

[1] Th. Andreae. Über maximale Systeme von kantendisjunkten unendlichen Wegen in Graphen. Math. Nachr., 101:219-228, 1981.

[2] R. Diestel. The cycle space of an infinite graph. Comb., Probab. Comput., 14:59-79, 2005.

[3] R. Diestel. Graph Theory (3rd ed.). Springer-Verlag, 2005.

[4] R. Diestel and D. Kühn. On infinite cycles I. Combinatorica, 24:69-89, 2004.

[5] R. Diestel and D. Kühn. On infinite cycles II. Combinatorica, 24:91-116, 2004.

[6] R. Diestel and D. Kühn. Topological paths, cycles and spanning trees in infinite graphs. Europ. J. Combinatorics, 25:835-862, 2004.

[7] G.A. Dirac. Some theorems on abstract graphs. Proc. London Math. Soc., 2:69-81, 1952. 
[8] R. Halin. A note on Menger's theorem for infinite locally finite graphs. Abh. Math. Sem. Univ. Hamburg, 40:111-114, 1974.

[9] D.W. Hall and G.L. Spencer. Elementary Topology. John Wiley, New York 1955.

[10] F. Laviolette. Decompositions of infinite graphs: Part II - Circuit decompositions. Preprint 2003.

[11] W. Mader. Homomorphieeigenschaften und mittlere Kantendichte von Graphen. Math. Ann., 174:265-268, 1967.

[12] C.St.J.A. Nash-Williams. Decomposition of graphs into two-way infinite paths. Can. J. Math., 15:479-485, 1963.

Version 06 Aug 2005

Henning Bruhn <hbruhn@gmx.net>

Maya Stein <fm7y052@public.uni-hamburg.de>

Mathematisches Seminar

Universität Hamburg

Bundesstraße 55

20146 Hamburg

Germany 